\title{
A bioinformatics approach to identify telomere sequences
}

\author{
Indira Somanathan ${ }^{1} \&$ Chris Baysdorfer ${ }^{\star, 1}$ \\ ${ }^{1}$ Department of Biological Sciences, California State University East Bay, Hayward, CA, USA
}

BioTechniques 65: 20-25 (July 2018) 10.2144/btn-2018-0057

Keywords: bioinformatics $\bullet$ next-generation sequencing $\bullet$ NGS $\bullet$ sequence repeat finder $\bullet$ SERF $\bullet$ tandem repeats $\bullet$ telomere

Conventional approaches to identify a telomere motif in a new genome are laborious and time-intensive. An efficient new methodology based on next-generation sequencing (NGS), de novo sequence repeat finder (SERF) and fluorescence in situ hybridization (FISH) is presented. Unlike existing heuristic approaches, SERF utilizes an exhaustive analysis of raw NGS reads or assembled contigs for rapid de novo detection of conserved tandem repeats representing telomere motifs. SERF was validated using the NGS data from Ipheion uniflorum and Allium cepa with known telomere motifs. The analysis program was then used on NGS data to investigate the telomere motifs in several additional plant species and together with FISH proved to be an efficient approach to identify as yet unknown telomere motifs.

\section{Introduction}

Eukaryotic linear chromosome ends are characterized by telomeres, which are generally made up of long tracts of minisatellite tandem repeats [1]. Telomeres are maintained by telomerase, a reverse transcriptase-based end maintenance system [2]. Telomeres help to prevent the loss of essential genetic information from the chromosome ends resulting from the incomplete replication of the lagging strand of chromosomal DNA and help to avoid detrimental chromosomal end-to-end fusions by distinguishing the double-stranded DNA (dsDNA) breaks from the native ends of the chromosomes [3]. Telomeres, together with their associated proteins, secure and stop the degradation of the chromosomal ends by various nucleolytic enzymes, as reviewed in de Lange [4]. Thus, telomeres maintain the integrity and stability of chromosomes.

The telomere repeat motif (TTTAGGG), discovered in Arabidopsis thaliana [5], is considered to be the consensus telomere motif for most land plants [6]. Similarly, other groups of organisms have shown conservation of their telomere motifs. However, there are a few exceptions to the ancestral-type consensus telomere motif. Drosophila melanogaster, a dipteran, lacks telomerase and the insect-type telomere repeat motif $($ TTAGG) $[7,8]$. Its telomeres have been replaced by three different types of telomere-specific non-long terminal repeat (non-LTR) retrotransposons [9], namely, HeT-A [10], TART [11] and TAHRE [12], which perform the telomere functions. In various species of algae, telomere repeat motif sequences are seen to differ slightly from the land plant telomere motif (TTTAGGG) [13]. For example, in the green alga Chlamydomonas reinhardtii, a more T-rich telomere repeat motif (TTTTAGGG) ${ }_{n}$ has been detected [14]. A telomere repeat motif switch identical to that found in Chlamydomonas-type was also seen in a few species of algae from the genus Klebsormidium [15,16].

Telomere motif evolution has also been demonstrated across various groups of land plants [17]. The Arabidopsis-type telomere is lost in Cestrum elegans from the family Solanaceae [18] and is replaced by an unusual 10 base pair (bp) telomere motif (TTTTTAGGG) maintained by telomerase [19]. Yet, many model plant species for telomere studies from this family, such as Nicotiana tabacum, Solanum lycopersicum and Solanum tuberosum, carry the ancestral-type telomere motif(TTTAGGG) ${ }_{n}$ at their chromosome termini [20-22]. Telomere divergence has also been shown to occur in monocots, especially in the order Asparagales. In the family Asphodelaceae, plants of the genus Aloe lack the Arabidopsis-type (TTTAGGG) telomere motif [23] and instead have the human-type (TTAGGG) [24]. Different types of telomere motifs are discovered in the family Amaryllidaceae. For example, in /pheion uniflorum, the canonical Arabidopsis-type telomere motif is replaced by human-type [25], while in Allium cepa and related species, a unique 12 base pair telomere motif (CTCGGTTATGGG) [26] has replaced both the Arabidopsis-type (TTTAGGG) [27,28], and the human-type (TTAGGG) $[25,29]$ telomere motifs. It took more than a decade of extensive research to finally identify the distinct telomere motifs in the genera Cestrum and Allium.

\section{METHOD SUMMARY}

Here we introduce a bioinformatics-based methodology for the rapid de novo detection of the most probable telomere motifs in genomes of any size. Raw unassembled genomic data from next-generation sequencers are analyzed using the newly developed SERF de novo sequence analysis algorithm to identify a culled set of putative telomere motifs based on their biological characteristics. The cytological validation of these motifs using fluorescence in situ hybridization followed by its detection using confocal microscopy can expedite the identification of true telomere sequence with fewer wet-lab iterations. 
A number of specific approaches to quantify human telomere length [30] from whole genome squencing (WGS) data use the known telomere motif (TTAGGG). The few de novo repeat detection tools [31-33] available are not equipped with a probable telomere motif detection algorithm from the NGS sampling data or WGS data. Consequently, efforts to detect telomere motifs employ time-intensive laboratory protocols and complex computational methods [26,34].

Future efforts to identify as yet unknown telomere motifs in a variety of genomes can be expedited using bioinformatics. We present a simple and efficient methodology that utilizes NGS data in conjunction with sequence repeat finder (SERF) algorithms, which is tailored towards identifying the probable conserved telomere repeat motifs and their subsequent cytological validation using fluorescence in situ hybridization (FISH) [35] (Figure 1).

\section{Materials \& methods}

\section{Plant materials}

Plant materials used in this research include Ipheion uniflorum, Allium cepa, Narcissus jonquilla and Muscari comosum, obtained from commercial sources.

Isolation of total genomic DNA

Total genomic DNA was isolated from young leaves of these plants using the Qiagen DNeasy plant mini kit (Hilden, Germany) as per manufacturer's protocols. Approximately $100 \mathrm{mg}$ (wet weight) of the leaf tissue was used for each of the isolations.

\section{Next-generation sequencing}

Genome sampling data was collected in multiple runs from A. cepa (GenBank Sequence Read Archive [SRA] accession numbers: SRX3381812, SRX3381811 and SRX3381814) and N. jonquilla (GenBank SRA accession numbers: SRX3381816 and SRX3381815) and in single runs from I. uniflorum (GenBank SRA accession number: SRX3381813) and M. comosum (GenBank SRA accession number: SRX3381817) using the lon Torrent nextgeneration sequencing (NGS) platform (Life Technologies, CA, USA), as per manufacturer's instructions. De novo assemblies for A. cepa and I. uniflorum were performed using the software SegMan NGen (www. dnastar.com). Previously sampled plant genomes from more than 20 species belonging to the families Solanaceae, Liliaceae, Smilacaceae and Velloziaceae (Unpublished Data) were also used to validate the telomere motif identification methodology (see Table 4).

\section{SERF de novo telomere}

sequence analysis

Consensus sequences from the whole genome assemblies in FASTA format and the raw NGS reads data in FASTQ format were separately analyzed by the sequence repeat finder (SERF) analysis platform (bioserf.org) to de novo identify and extract contiguous conserved tandem repeats varying in size from 5-15 base pairs. Genomes of 1 . uniflorum with human-type (TTAGGG) [25] and $A$. cepa with a unique 12 base pair (CTCGGTTATGGG) [26] telomere repeat motifs were used as positive controls for the initial validation of the SERF algorithm.

Homopolymeric nucleotide tracts and supersets of repeats were filtered out from the analysis results. This analysis looked for a pattern of organization (contiguous versus scattered), positional distribution of the repeats within the contigs or reads, and the frequency of the conserved repeat motifs in these sequences. The quality metric concept employed in SERF reflected the percentage of the individual read or contig length occupied by the identified contiguous repeats. A similar approach was used in A. cepa telomere motif identification [26]. Permutative combinations were analyzed, and repeats were clustered based on the size, position and type of base pair. Based on the assumptions that telomeres are poorly represented in genome sampling, and that the reads generated using the lon

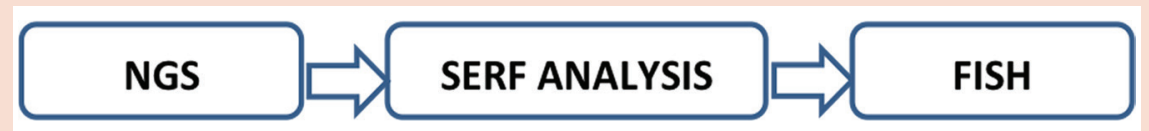

Figure 1. Workflow: NGS-SERF-based approach. Putative telomere repeat motif analysis by SERF using the genomic NGS data as raw reads (FASTQ) or contig assembly (FASTA) followed by cytological validation with FISH.

FISH: Fluorescence in situ hybridization; NGS: Next-generation sequencing; SERF: Sequence repeat finder.
Torrent are generally short in length, an initial protocol of 2-5-15-90-TG-contiguous (minimum number of contiguous repeatsminimum base pair size-maximum base pair size-90\% quality metric-TG richnesscontiguous mode) was employed. Emphasis was given to the quality metric rather than the number of tandem telomere motifs to analyze the assembled contigs in l.uniflorum. An identical protocol successfully identified the respective telomere motifs from unassembled NGS reads of $I$. uniflorum and $A$. cepa with a high quality metric. The same protocol was used on all other species tested. Additional information on SERF can be found in the Supplementary material (Figure S1) and from bioserf.org.

Probe synthesis for FISH on $N$. jonquilla

11-dUTP biotinylated probes were synthesized using PCR with $N$. jonquilla genomic DNA for $(A)$ the unique $15 \mathrm{bp}$ (GAGGGTGCACTCACG) ${ }_{n}$ and (B) humantype (TTAGGG) ${ }_{n}$ repeats identified by SERF analysis on its raw NGS reads. The following primers were used: for repeat (A): 5' ACTCACGGAGGGTGCACTCACG 3' (for), 5' CGTGAGTGCACCCTCCGTGAGT 3' (rev), and for repeat (B): 5' TTAGGGTTAGGGTTAGGGTTAGGG 3' (for) and 5' CCCTAACCCTAACCCTAACCCTAA 3' (rev). The lengths of the probes were between 200 and $400 \mathrm{bp}$.

Preparation of metaphase chromosome spreads of $N$. jonquilla Young root tips (3-5 days old) from $N$. jonquilla bulbs were arrested in metaphase by ice cold water treatment, fixed in freshly prepared Carnoy's solution I, and spread by squashing [36]. Metaphase chromosome spreads on slides were immediately frozen at $-80^{\circ} \mathrm{C}$ for $5-10 \mathrm{~min}$ before they were fixed in $4 \%$ formaldehyde in 2XSSC (saline sodium citrate) for $10 \mathrm{~min}$. Slides were washed twice (5 min each) in 2XSSC followed by $95 \%$ ethanol fixation for another $10 \mathrm{~min}$. Slides were air-dried, and those with good metaphase spreads were selected for performing FISH.

In situ hybridization on N. jonquilla metaphase chromosome spreads A slightly modified version of the FISH protocol by Fajkus et al. [26] was followed. First, $30 \mu$ l/slide of hybridization mix, which included $50 \%(\mathrm{~V} / \mathrm{v})$ deionized formamide (Applied Biosystems, USA), 10\% (w/v) dextran sulphate in 2×SSC, 200-300 ng 
of the biotinylated probe, $50 \times$ salmon sperm carrier DNA (stock 10 mg/ml; 5 Prime -> 3 Prime, Inc., CO, USA) was prepared. The chromosomal DNA on the slides, together with the probe in the hybridization mixture, was denatured in a thermocycler for $3 \mathrm{~min}$ at $80^{\circ} \mathrm{C}$. The slides were promptly moved to a moist chamber kept at $35^{\circ} \mathrm{C}$ in a hybridization chamber and hybridized overnight for 12-16 h. The cover slip was carefully removed by dipping in $2 \times S S C$ at $42^{\circ} \mathrm{C}$. Posthybridization washes were comprised of 3 $\times 5$ min each at $42^{\circ} \mathrm{C}$ in $40 \%$ formamide in $2 \times \mathrm{SSC}, \mathrm{pH} 7 ; 1 \times 5 \mathrm{~min}$ in $2 \times \mathrm{SSC}$ at $42^{\circ} \mathrm{C}$; $1 \times 10 \mathrm{~min}$ in $2 \times \mathrm{SSC}$ at room temperature. Slides were incubated with streptavidin 680-I dye conjugate (Abnova, Taiwan) at 1:200 dilution in $2 \times S S C$ for 40 min in a $37^{\circ} \mathrm{C}$ moist chamber and excess conjugate mix was washed off in $2 \times S S C$ at $45^{\circ} \mathrm{C}$ for $10 \mathrm{~min}$. Finally, the slides were air-dried and counterstained with ProLong ${ }^{\circledR}$ Diamond Antifade Mountant with DAPI (4',6-diamidino-2 phenylindole) by Life Technologies. Hybridization signals were imaged using a Leica TCS SP8 Confocal Microscope (Wetzlar, Germany).

\section{Results \& discussion}

ASERF protocol of 2-5-15-90-TG-contiguous was used to validate the human-type telomere motif from the unassembled NGS reads and contig assembly of I. uniflorum. SERF analysis successfully identified its telomere motif despite the fact that only 1.4\% (0.13 Gbp/9.44 Gbp) of the genome was available for analysis (Figure 2).

Table 1 shows the identification of the human-type telomere motif from the contig assembly of 1 . uniflorum. The analysis retrieved a single short contig of 59 base pairs in length consisting of 14 reads and harboring nine of the human-type (TTAGGG) telomere repeat motifs in tandem with a high quality metric of $91 \%$. SERF analysis on the unassembled NGS reads of $I$. uniflorum has also detected an identical repeat motif output from multiple reads with high quality metric (data not shown).

SERF analysis detected and retrieved a single contig of 39 base pairs from $A$. cepa's contig assembly of the pooled read sets with the known Allium telomere repeat motif (Table 2). The identified telomere motif from the assembled contig has a high quality metric of $92 \%$ and is rich in $T$ and $G$ as well. The telomere repeat motif in its various permutative forms was also located in many

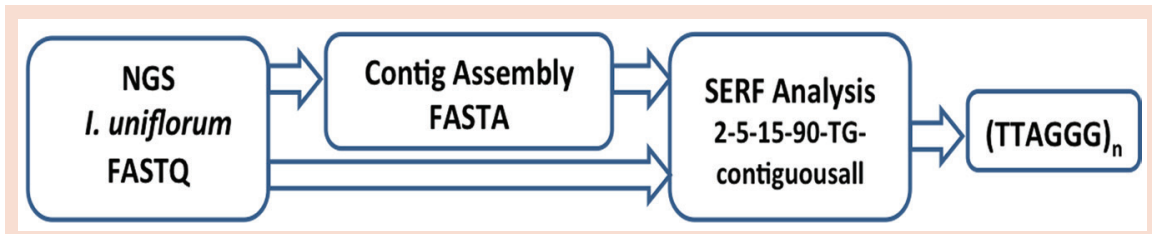

Figure 2. Telomere motif detection of Ipheion uniflorum with NGS-SERF-based approach. Detection of the human-type (TTAGGG) telomere repeat motif in $I$. uniflorum from its raw NGS reads and contig assembly using SERF.

NGS: Next-generation sequencing; SERF: Sequence repeat finder.

reads of single $A$. cepa NGS runs using the same protocol (Table 3). Depending on the number of NGS reads that harbor these extended repeats, assembly may not always yield a contig with these repeat motifs, which makes analysis on unassembled NGS data more desirable. WGS can overcome this difficulty and can generate a sufficient number of reads to form contig representing the telomere, enabling the measurement of the telomere content in the genome $[30,37]$.

Sample output of the SERF analysis on one of the raw NGS sampling data sets of $A$. cepa using the protocol 2-5-15-90-TG-contiguous for a selected quality metric of $90 \%$ (Figure S2), FISH on its metaphase chromosome spreads (Figure S3) and the FISH methods are included in the Supplementary Material.

An extensive study of telomere repeat motif identification was also undertaken on the unassembled NGS data from genomic sampling of over 20 plant species from diverse families such as Amaryllidaceae, Hyacinthaceae, Liliaceae, Solanaceae, Smilacaceae and Velloziaceae (Table 4). Many of the plants analyzed have previously identified or probable consensus telomere repeat motifs of their respective families or genera. SERF analysis on $F$. imperialis predominantly detected the presence of Arabidopsis-type telomere repeat motifs, but combination repeats such as TTTAGGGTTAGGG and TTTAGGGTTTAGGGG were also detected, but with a lower quality metric (44 and 54\%). SERF analysis also identified both human-type (TTAGGG) $)_{n}$ and Bombyx-type (TTAGG) putative telomere motifs from a single read in F. pudica (41 and 48\%) and Arabidopsis-type and Bombyx-type as putative telomere motifs in T. nana (100 and 98\%). Analysis of Xerophyta humilis from the family Velloziaceae identified (TTTAGGG) and (TTTTAGG) ${ }_{n}$ as probable telomere repeat motifs with a high-quality metric (100 and 84\%). The probable telomere repeat motifs (Table 4, superscript 5) identified by SERF analysis from various species require further cytological confirmation. Most species that were analyzed had unassembled NGS data equivalent to 50-150 Mbp and were processed by SERF in a few hours.

$\ln N$. jonquilla and $M$. comosum from the order Asparagales, the Arabidopsis-type and human-type telomere repeat motifs were originally shown to be absent from their genome [38]. Later studies, however, did suggest the presence of these two types of telomere repeat motifs with an abundance of the human-type in Narcissus triandrus and Muscari neglectum telomeres [39]. SERF analysis on $N$. jonquilla's raw NGS data sets identified a 15 bp unique conserved tandem repeat motif (GAGGGTGCACTCACG) on a read with a high quality metric (90\%) in addition to the human-type telomere repeat motif (TTAGGG) with 90\% (Table 5). Higher probability of human-type motif as $N$. jonquilla telomere motif is evident from its abundance in the PCR amplification products (Figure S4). Analysis of the raw NGS data of $M$. comosum also revealed the abundance of human-type telomere repeat motif in multiple reads (Table 4). A few Arabidopsis-type repeats in tandem were also retrieved, but with a low quality metric.

The human-type and unique repeats identified by SERF analysis were subjected to cytological validation on $N$. jonquilla metaphase chromosome spreads using FISH. FISH with probes synthesized using the repeat motif (GAGGGTGCACTCACG) did not yield any telomere-specific signals (data not shown), whereas FISH performed using probes specific to the humantype telomere repeat motif (TTAGGG) generated clear signals from the telomeres on the metaphase chromosome spreads of $\mathrm{N}$. jonquilla (Figure 3 ).

Telomere repeat motifs identified by SERF from the analysis of plant genomes (Table 4) are deposited in the SERFDB database (bioserf.org). Some motifs already have cytological validation from previous work, while others require such validation. 
Table 1. Telomere motif identification of Ipheion uniflorum from the contig assembly.

\begin{tabular}{|c|c|c|c|c|c|c|}
\hline $\begin{array}{l}\text { Order } \\
\text { Family } \\
\text { Species }\end{array}$ & $\begin{array}{l}\text { Telomere type \& } \\
\text { motif }\end{array}$ & No. of base pairs & $\begin{array}{l}\text { No. of tandem } \\
\text { repeats }\end{array}$ & $\begin{array}{l}\text { Length of contig } \\
\text { tandem }\end{array}$ & $\begin{array}{l}\text { No. of reads with } \\
\text { tandem repeats }\end{array}$ & SERF protocol \\
\hline $\begin{array}{l}\text { Asparagales } \\
\text { Amaryllidaceae } \\
\text { 1. uniflorum }\end{array}$ & Human-type TTAGGG & 6 & 9 & 59 & 14 & $\begin{array}{l}\text { 2-5-15-90-TG- } \\
\text { contiguous }\end{array}$ \\
\hline
\end{tabular}

Table 2. Identification of $A$. cepa telomere repeat motif from the contig assembly.

\begin{tabular}{|c|c|c|c|c|c|c|}
\hline $\begin{array}{l}\text { Order } \\
\text { Family } \\
\text { Species }\end{array}$ & $\begin{array}{l}\text { Telomere type \& } \\
\text { motif }\end{array}$ & No. of base pairs & $\begin{array}{l}\text { No. of tandem } \\
\text { repeats }\end{array}$ & $\begin{array}{l}\text { Length of contig } \\
\text { tandem }\end{array}$ & $\begin{array}{l}\text { No. of reads with } \\
\text { tandem repeats }\end{array}$ & SERF protocol \\
\hline $\begin{array}{l}\text { Asparagales } \\
\text { Amaryllidaceae } \\
\text { A. cepa }\end{array}$ & $\begin{array}{l}\text { Unique-type } \\
\text { TGGGCTCGGTTA }\end{array}$ & 12 & 3 & 39 & 9 & $\begin{array}{l}\text { 2-5-15-90-TG- } \\
\text { contiguous }\end{array}$ \\
\hline
\end{tabular}

The majority of land plants were initially thought to have the Arabidopsis-type telomere motifs at their chromosome termini, but the universality of this ancestral motif came under question once its absence from telomeres had been detected in monocots from the families Amaryllidaceae (earlier known as Alliaceae) [28] and Asphodelaceae [23], both belonging to the order
Asparagales. Fuch et al. (1995) [28] showed that plants in the genus Allium from the Amaryllidaceae family have completely lost the typical ancestral-type telomere repeat motif (TTTAGGG) from both telomeres and interstitial regions. These evolutionary switches in telomere motif sequence have been widespread in the order Asparagales and the typical consensus plant telomere motifs are replaced in many plants with unique repeat sequences that were never before detected [26].

Conventional methods to find an unknown telomere motif in a new genome are challenging. A positive outcome results only if the organism under investigation maintains one (or a combination) of the known consensus telomere motifs in its

Table 3. Identification of $A$. cepa telomere repeat motif from unassembled NGS reads.

\begin{tabular}{l|l|l|l|}
$\begin{array}{l}\text { Order } \\
\text { Family } \\
\text { Species }\end{array}$ & Telomere type and motif & No. of base pairs & No. of tandem repeats \\
\hline $\begin{array}{l}\text { Asparagales } \\
\text { Amaryllidaceae } \\
\text { A. cepa }\end{array}$ & Unique-type TGGGCTCGGTTA & 12 & 3 \\
\hline $\begin{array}{l}\text { Motif identified by SERF and the corresponding read containing the motif: TCGGTTATGGGC I rep 3 I size 12 I pos 1 I qual 92\%@F3UVD:00611:00677 TCGGTTATGGGCTCGGTTATGGGCTCGGT- } \\
\text { TATGGGCTCGTGGCTCGGTTA I rep 3 I size 12 I pos 1 I qual 94\%@F3UVD:00617:00049TGGGCTCGGTTATGGGCTCGGTTATGGGCTCGGTTATG Identification of } \text { A. cepa telomere motif with a size of } \\
\text { 12 bp in its permutative forms in the raw reads from one of the unassembled NGS read sets using the protocol 2-5-15-90-TG-contiguous. } \\
\text { NGS: Next-generation sequencing; SERF: Sequence repeat finder. }\end{array}$ \\
\hline
\end{tabular}

Table 4. SERF telomere motif investigation on additional plant species.

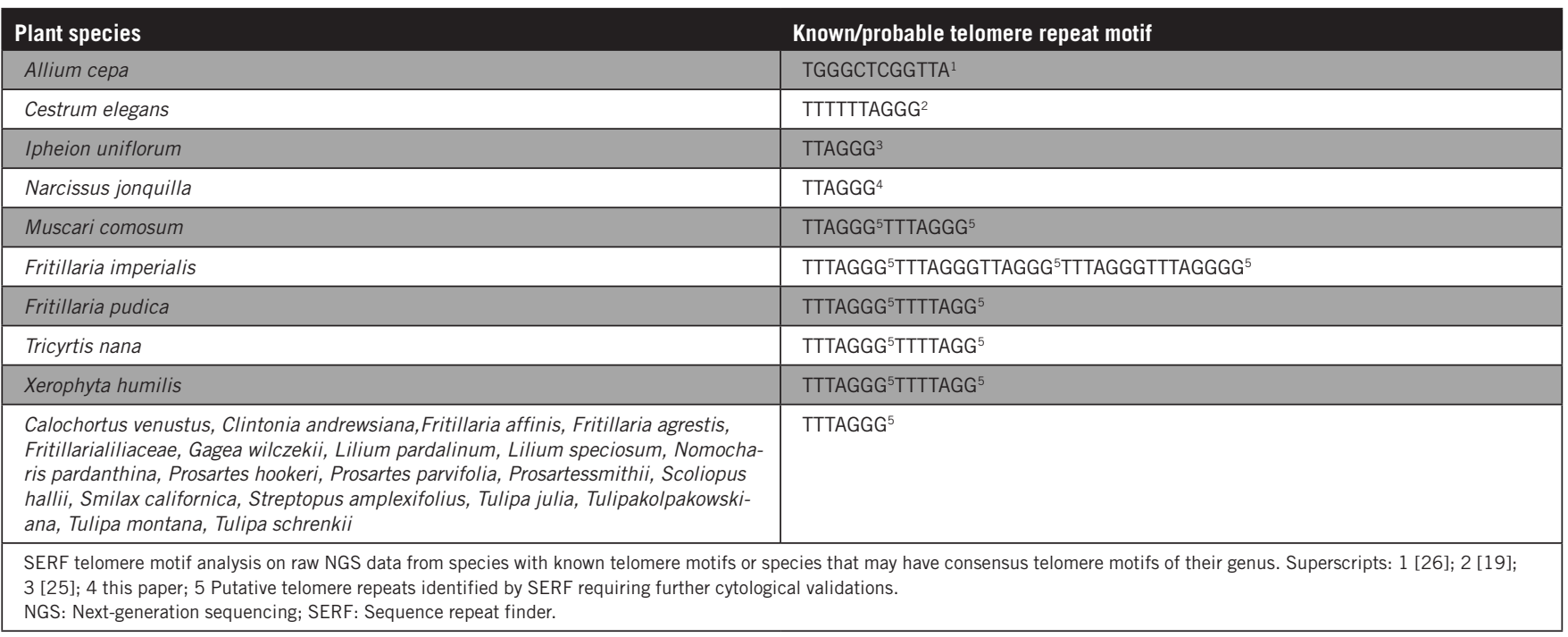


Table 5. Probable telomere motifs identified from the raw NGS data of $N$. jonquilla.

\begin{tabular}{|c|c|}
\hline $\begin{array}{l}\text { Order } \\
\text { Family } \\
\text { Species }\end{array}$ & SERF protocol \\
\hline $\begin{array}{l}\text { Asparagales } \\
\text { Amaryllidaceae } \\
\text { N. jonquilla }\end{array}$ & 2-5-15-90-TG-contiguous \\
\hline \multicolumn{2}{|c|}{$\begin{array}{l}\text { Motif identified by SERF and the corresponding read containing the motif: GGGTTA I rep } 8 \text { I pos } 1 \text { I size } 6 \text { I bases } 53 \text { I qual 90\%@S9N6T:00060:01055GGGTTAGGGTTAGGGTTAGGGTTAGG } \\
\text { GTTAGGGTTAGGGTAGGGTTAGGTTAGAGGGTGCACTCACG I rep } 6 \text { I pos } 1 \text { I size } 15 \text { I bases } 100 \text { I qual } 90 \% \text { @ } 7 \text { ZXN9:00683:00043 GAGGGTGCACTCACGGAGGGTGCACTCACGGAGGGTGCACTCACG- } \\
\text { GAGGGTGCACTCACGGAGGGTGCACTCACGGAGGGTGCACTCACGAGGGATGCAA Two potential telomere motifs of } N \text {. jonquilla identified from the unassembled NGS read sets using the protocol } \\
\text { 2-5-15-90-TG-contiguous. One was the human-type and the other one was a unique motif of } 15 \text { bp. } \\
\text { NGS: Next-generation sequencing; SERF: Sequence repeat finder. }\end{array}$} \\
\hline
\end{tabular}

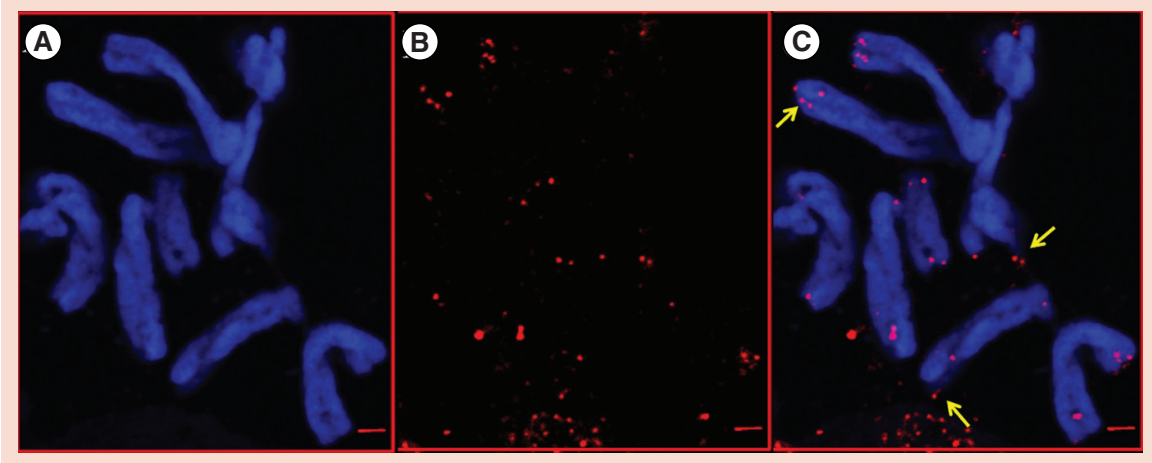

Figure 3. FISH signals from the telomeres of $N$. jonquilla metaphase chromosomes hybridized with human-type telomere probes. (A) Chromosomes (blue) counterstained with DAPI. (B) Signal from the hybridized telomere probes detected with streptavidin 680-I dye conjugate (red). (C) Merged image of (A) and (B). Arrows point to the telomere probe hybridization signals. The scale is $2.01 \mu \mathrm{m}$.

chromosome termini. The recent detection of novel telomere motifs, especially in the order Asparagales [26], illustrates the challenge and warrants the need to switch from the conventional approach to one that utilizes modern sequencing technology and analysis platforms for an effective utilization of available resources. Such a shift is occurring and is seen in the discovery of the telomere motifs of $C$. elegans [19] and A. cepa $[26,34]$.

The SERF analysis platform utilized here employs multiple features to increase its accuracy and efficiency in telomere repeat motif identification. The selection of tandem repeats rich in T and G [14], enabled the elimination of an inordinate number of irrelevant tandem repeat motifs. As guaninerich telomere motifs are a hallmark of telomeres $[40,41]$, removal of repeats without a G-rich region was important in the telomere motif search. The introduction of the quality metric facilitated identification of tandem repeat motifs with telomere characteristics using the SERF protocol (2-5-15-90\%-TG-contiguous). Additional features such as clustering, identification of permutations, homopolymeric tracts and supersets, helped in the filtering of an overwhelming number of repeats that otherwise would have crowded the analysis results. These specific search attributes resulted in the successful identification of telomere motifs from both contig assembly and raw NGS reads. Use of I. uniflorum and $A$. cepa with known telomere motifs as positive controls enabled the fine-tuning of the SERF algorithm to identify the most probable telomere motifs from any genome of interest.

Telomere repeat motif analysis on the positive control plants emphasizes the need to rely more on raw NGS reads rather than on the genome assemblies due to assembly process excluding some repeated sequences and contigs with low read depth. Hence, SERF analysis primarily utilized the raw NGS data on all of the additional species investigated here. The analysis of multiple species (Table 4) has either reinforced earlier findings [29,38,39,42,43] or identified other potential telomere repeat motifs bioinformatically, requiring further cytological confirmation.

In the standard SERF protocol (2-5-15-90-TG-contiguous), the quality metric of $90 \%$ successfully identified the known or most probable telomere motif from the majority of the plants studied. However, in a few instances variations in quality metric were required to address sequencing errors in the raw NGS reads and when the repeat motifs identified were longer, as in the case of F. imperialis, C. elegans and A. cepa
(Table 4). Our method enabled the rapid detection of two potential telomere repeat motifs from $N$. jonquilla and cytological validation confirmed that only the humantype repeat is present at the telomeres (Figure 3).

This research has demonstrated that the discovery of probable conserved telomere repeat motifs in any genome can be expedited by using SERF de novo tandem repeat analysis of the raw NGS data, followed by cytological validation using $\mathrm{FISH}$. This methodology is cost efficient and can be used for the rapid detection of as yet unknown telomere motifs in genomes.

\section{Author contributions}

IS and CB conceived and developed the NGS, SERF and FISH based approach. IS and CB performed genomic DNA extraction and NGS. IS performed SERF analysis and FISH-based cytological validation of the approach. IS wrote the paper and CB supervised the study and revised the manuscript.

\section{Acknowledgements}

This research would not have been possible without the NGS sequencing data from CSU East Bay Biocore Lab and the SERF analysis platform from bioserf.org. We acknowledge and thank them for their valuable support.

\section{Financial \& competing interests disclosure}

The authors have no relevant affiliations or financial involvement with any organization or entity with a financial interest in or financial conflict with the subject matter or materials discussed in the manuscript. This includes employment, consultancies, honoraria, stock ownership or options, expert testimony, grants or patents received or pending, or royalties. No writing 
assistance was utilized in the production of this manuscript.

\section{Open access}

This work is licensed under the Creative Commons Attribution 4.0 License. To view a copy of this license, visit http://creativecommons.org/licenses/by/4.0/

\section{Supplementary data}

To view the supplementary data that accompany this paper please visit the journal website at: www.future-science. com/doi/suppl/10.2144/btn-2018-0057

\section{References}

1. Blackburn EH, Gall JG. A tandemly repeated sequence at the termini of the extrachromosomal ribosomal RNA genes in Tetrahymena. J. Mol. Biol. 120(1), 33-53 (1978).

2. Greider $\mathrm{CW}$, Blackburn EH. Identification of a specific telomere terminal transferase activity in Tetrahymena extracts. Cell 43(2), 405-413 (1985).

3. Greider CW. Telomeres, telomerase and senescence. Bioessays 12(8), 363-369 (1990).

4. de Lange T. Shelterin: the protein complex that shapes and safeguards human telomeres. Genes Dev. 19(18), 2100-2110 (2005).

5. Richards EJ, Ausubel FM. Isolation of a higher eukaryotic telomere from Arabidopsis thaliana. Cell 53(1), 127-136 (1988).

6. Frydrychova RC, Mason JM. Telomeres: their structure and maintenance. In: The Mechanisms of DNA Replication. David Stuart (Ed.). InTech Publishing, London, UK, 423-443 (2013).

7. Okazaki S, Tsuchida K, Maekawa H, Ishikawa $H$, Fujiwara $H$. Identification of a pentanucleotide telomeric sequence, (TTAGG) $n$, in the silkworm Bombyx mori and in other insects. Mol. Cell. Biol. 13(3), 1424-1432 (1993).

8. Sahara K, Marec F, Traut W. TTAGG telomeric repeats in chromosomes of some insects and other arthropods. Chromosome Res. 7(6), 449460 (1999).

9. Pardue M-L, DeBaryshe PG. Retrotransposons that maintain chromosome ends. Proc. Natl Acad. Sci. USA 108(51), 20317-20324 (2011).

10. Biessmann H, Mason JM, Ferry Ket al. Addition of telomere-associated HeT DNA sequences 'heals' broken chromosome ends in Drosophila. Cell 61(4), 663-673 (1990).

11. Levis RW, Ganesan R, Houtchens K, Tolar LA, Sheen F. Transposons in place of telomeric repeats at a Drosophila telomere. Cell 75(6), 1083-1093 (1993).

12. Abad JP, de Pablos B, Osoegawa K et al. TAHRE, a novel telomeric retrotransposon from Drosophila melanogaster, reveals the origin of Drosophila telomeres. Mol. Biol. Evol. 21(9), 1620-1624 (2004).

13. Procházková Schrumpfová P, SchoŘová Š, Fajkus J. Telomere- and telomerase-associated proteins and their functions in the plant cell. Front. Plant Sci. 7, 851 (2016).
14. Petracek ME, Lefebvre PA, Silflow CD, Berman J. Chlamydomonas telomere sequences are $\mathrm{A}+$ T-rich but contain three consecutive G-C base pairs. Proc. Natl Acad. Sci. USA 87(21), 8222-8226 (1990).

15. Fulnecková J, Sevcíková T, Fajkus J et al. A broad phylogenetic survey unveils the diversity and evolution of telomeres in eukaryotes. Genome Biol. Evol. 5(3), 468-483 (2013)

16. FulneČková J, ŠevČíková T, Lukešová $A$, Sýkorová E. Transitions between the Arabidopsis-type and the human-type telomere sequence in green algae (clade Caudivolvoxa, Chlamydomonadales). Chromosoma 125(3), 437-451 (2016)

17. Fajkus J, Sýkorová E, Leitch AR. Telomeres in evolution and evolution of telomeres. Chromosome Res. 13(5), 469-479 (2005).

18. Sykorova E, Lim KY, Chase MW et al. The absence of Arabidopsis-type telomeres in Cestrum and closely related genera Vestia and Sessea (Solanaceae): first evidence from eudicots. Plant J. 34(3), 283-291 (2003).

19. Peška $\vee$, Fajkus $P$, Fojtová $M$ et al. Characterisation of an unusual telomere motif (TTTTTTAGGG)n in the plant Cestrum elegans (Solanaceae), a species with a large genome. Plant J. 82(4), 644-654 (2015).

20. Broun P, Ganal MW, Tanksley SD. Telomeric arrays display high levels of heritable polymorphism among closely related plant varieties. Proc. Natl Acad. Sci. USA 89(4), 1354-1357 (1992).

21. Fajkus J, Novotná M, Ptacek J. Analysis of chromosome termini in potato varieties. Rostl. Vyroba 48(11), 477-479 (2002).

22. Ganal MW, Lapitan NL, Tanksley SD. Macrostructure of the tomato telomeres. Plant Cell 3(1), 87-94 (1991).

23. Adams SP, Leitch IJ, Bennett MD, Leitch AR. Aloe L.- a second plant family without (TTTAGGG) n telomeres. Chromosoma 109(3), 201-205 (2000).

24. Weiss H, Scherthan H. Aloe spp. - plants with vertebrate-like telomeric sequences. Chromosome Res. 10(2), 155-164 (2002).

25. Sýkorová E, Fajkus J, Mezníková M et al. Minisatellite telomeres occur in the family Alliaceae but are lost in Allium. Am. J. Bot. 93(6), 814823 (2006).

26. Fajkus P, Peška V, Sitová Z et al. Allium telomeres unmasked: the unusual telomeric sequence (CTCGGTTATGGG)n is synthesized by telomerase. Plant J. 85(3), 337-347 (2016).

27. Pich U, Fuchs J, Schubert I. How do Alliaceae stabilize their chromosome ends in the absence of TTTAGGG sequences? Chromosome Res. 4(3), 207-213 (1996).

28. Fuchs J, Brandes A, Schubert I. Telomere sequence localization and karyotype evolution in higher plants. Plant Syst. Evol. 196(3-4), 227241 (1995).

29. Sýkorová E, Lim KY, Kunická Z et al. Telomere variability in the monocotyledonous plant order Asparagales. Proc. R. Soc. Lond., B, Biol. Sci. 270(1527), 1893-1904 (2003).

30. Lee M, Napier CE, Yang SF, Arthur JW, Reddel RR, Pickett HA. Comparative analysis of whole genome sequencing-based telomere length measurement techniques. Methods 114, 4-15 (2017).
31. Girgis HZ. Red: an intelligent, rapid, accurate too for detecting repeats de-novo on the genomic scale. BMC Bioinformatics 16, 227 (2015).

32. Wirawan A, Kwoh CK, Hsu LY, Koh TH. INVERTER: INtegrated Variable numbER Tandem rEpeat findeR. In: Computational Systems-Biology and Bioinformatics. Chan JH, Ong YS, Cho SB (Eds). Springer, Berlin, Heidelberg, Germany, 151-164 (2010).

33. Novák P, Neumann P, Pech J, Steinhaisl J, Macas J. RepeatExplorer: a Galaxy-based web server for genome-wide characterization of eukaryotic repetitive elements from next-generation sequence reads. Bioinformatics 29(6), 792793 (2013).

34. Peška V, Sitová Z, Fajkus P, Fajkus J. BAL31-NGS approach for identification of telomeres de novo in large genomes. Methods 114, 16-27 (2017).

35. Somanathan I. Methods and Analytics to Identify Telomere Repeats in Genomes. M.S. Thesis. Biological Sciences Department, California State University East Bay, Hayward, CA, USA (2016).

36. Mirzaghaderi G. Simple metaphase chromosome preparation from meristematic root tip cells of wheat for karyotyping or in situ hybridization. Afr. J. Biotechnol. 9(3), 314-318 (2010).

37. Feuerbach L, Sieverling L, Deeg $\mathrm{K}$ et al. TelomereHunter: telomere content estimation and characterization from whole genome sequencing data. bioRxiv 065532 (2016).

38. de La Herrán R, Cuñado N, Navajas-Pérez R et al. The controversial telomeres of lily plants. Cytogenet. Genome Res. 109(1-3), 144-147 (2005).

39. Shibata F, Hizume M. Survey of Arabidopsis-and human-type telomere repeats in plants using fluorescence in situ hybridisation. Cytologia (Tokyo, Japan) 76(3), 353-360 (2011).

40. Williamson JR, Raghuraman MK, Cech TR. Monovalent cation-induced structure of telomeric DNA: the G-quartet model. Cell 59(5), 871-880 (1989).

41. Simonsson T. G-quadruplex DNA structures variations on a theme. Biol. Chem. 382(4), 621628 (2001)

42. Adams SP, Hartman TPV, Lim KY et al. Loss and recovery of Arabidopsis-type telomere repeat sequences $5^{\prime}$-(TTTAGGG) n-3' in the evolution of a major radiation of flowering plants. Proc. $R$. Soc. Lond., B, Biol. Sci. 268(1476), 1541-1546 (2001).

43. Fujimoto S, Ito M, Matsunaga S, Fukui K. An upper limit of the ratio of DNA volume to nuclear volume exists in plants. Genes Genet. Syst. 80(5), 345-350 (2005).

First draft submitted: 9 December 2018; Accepted for publication: 24 May 2018

Address correspondence to: Chris Baysdorfer; Department of Biological Sciences, California State University East Bay, Hayward, CA, USA; E-mail: chris. baysdorfer@csueastbay.edu

To purchase reprints of this article contact: s.cavana@future-science.com 